\title{
Simplified immunosuppressive and neuroprotective agents based on gracilin $A$
}

\author{
Mikail E. Abbasov1, Rebeca Alvariño $\mathbb{D}^{2}$, Christian M. Chaheine ${ }^{1}{ }^{1}$, Eva Alonso², Jon A.Sánchez²,

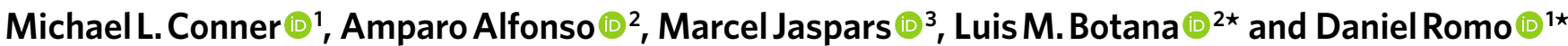

\begin{abstract}
The architecture and bioactivity of natural products frequently serve as embarkation points for the exploration of biologically relevant chemical space. Total synthesis followed by derivative synthesis has historically enabled a deeper understanding of structure-activity relationships. However, synthetic strategies towards a natural product are not always guided by hypotheses regarding the structural features required for bioactivity. Here, we report an approach to natural product total synthesis that we term 'pharmacophore-directed retrosynthesis'. A hypothesized, pharmacophore of a natural product is selected as an early synthetic target and this dictates the retrosynthetic analysis. In an ideal application, sequential increases in the structural complexity of this minimal structure enable development of a structure-activity relationship profile throughout the course of the total synthesis effort. This approach enables the identification of simpler congeners retaining bioactivity at a much earlier stage of a synthetic effort, as demonstrated here for the spongiane diterpenoid, gracilin A, leading to simplified derivatives with potent neuroprotective and immunosuppressive activity.
\end{abstract}

T he impactful and enduring role that natural products have played in improving the quality and duration of life for both humans and animals cannot be overstated. For example, rapamycin and its congeners have received Food and Drug Administration approval for various ailments, and this natural product continues to provide insights into basic cell biology ${ }^{1}$. Synthetic chemists are at the forefront of harvesting the full potential of natural products through synthetic efforts including classic total synthesis. Towards this goal, strategies to synthesize natural products have evolved significantly in recent years as more emphasis has been placed on biological function ${ }^{2}$. In Danishefsky's 'diverted total synthesis' (DTS), a synthetic sequence is developed from simple building blocks employing classical retrosynthetic analysis and then various advanced intermediates previously employed in the synthesis effort are diverted towards simplified derivatives for biological

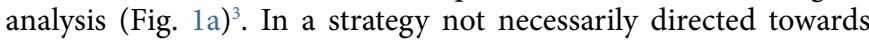
total synthesis, Wender's 'function-oriented synthesis' seeks to develop hypotheses regarding pharmacophores based on structural and computational analysis of distinct natural products and typically larger structure-activity relationship (SAR) data sets enabling the design and synthesis of simplified derivatives bearing a common pharmacophore ${ }^{4}$. Alternatively, Schreiber's 'diversity oriented synthesis' (DOS) of natural product-like libraries ${ }^{5}$ and the derived 'biology-oriented synthesis' (BIOS) by Waldmann ${ }^{6}$ seeks to synthesize collections of compounds based on structural features found in natural products. Finally, Myers recently developed a convergent building block strategy for rapid access to a diverse array of structurally related scaffolds such as macrolides related to erythromycin for the discovery of novel antibiotics ${ }^{7}$; this was subsequently termed 'analogue-oriented synthesis' by Vanderwal ${ }^{8}$.

The simultaneous alignment of total synthesis efforts with structure-activity relationship (SAR) studies has not been fully realized to the extent possible and in particular with novel natural products for which minimal SAR information exists. Several truncated natural products ${ }^{9}$, found to possess similar bioactivity to the parent natural product, are known (for example, eribulin mesylate from halichondrin ${ }^{10}$ ); however, these derivatives were typically identified following completion of a total synthesis ${ }^{11}$. In an example from our laboratory, a des-methyl, des-amino variant of the protein translation initiation inhibitor pateamine A was designed and synthesized following our total synthesis and found to have nearly equipotent activity to the natural product ${ }^{12}$. This led to a retrospective question of whether such a derivative may have been accessed en route to the natural product, leading us to pose the following question. Can the total synthesis of natural products, in particular with limited SAR or unknown or unconfirmed cellular targets, be more closely aligned to proposed biological activity during the retrosynthetic planning stages?

Here, we describe a type of retrosynthetic analysis that seeks to more closely align total synthesis efforts with concurrent biological studies. The strategy enables the identification of simplified versions of the natural product with similar potency or potentially new functions in the course of a total synthesis effort. We term this strategy 'pharmacophore-directed retrosynthesis' (PDR) to emphasize the importance of considering hypothesized pharmacophores at the retrosynthetic planning stage of a total synthesis effort. Although this approach increases the challenges of natural product total synthesis beyond important, contemporary goals including atom economy ${ }^{13}$, step and redox efficiency ${ }^{14}$, and protecting group avoidance ${ }^{15}$, it has the potential to greatly accelerate harvesting of the vast information content of natural products for basic cell biology and medicine. In PDR, we build on Wender's notion of bringing function to the forefront of a synthetic endeavour (function-oriented synthesis), but employ the logic of retrosynthesis ${ }^{16}$ to target simplified intermediates that importantly possess the proposed minimal structural features required for bioactivity (the pharmacophore) en route to the natural product ${ }^{17}$. In applying PDR, a key first step is identification of a proposed pharmacophore. This may be based on (1) structural

'Department of Chemistry and Biochemistry, Baylor University, Waco, TX, USA. 'Departamento de Farmacología, Facultad de Veterinaria, Universidad de Santiago de Compostela, Lugo, Spain. ${ }^{3}$ Marine Biodiscovery Centre, Department of Chemistry, University of Aberdeen, Aberdeen, Scotland, UK.

*e-mail: luis.botana@usc.es; Daniel_Romo@baylor.edu 
a

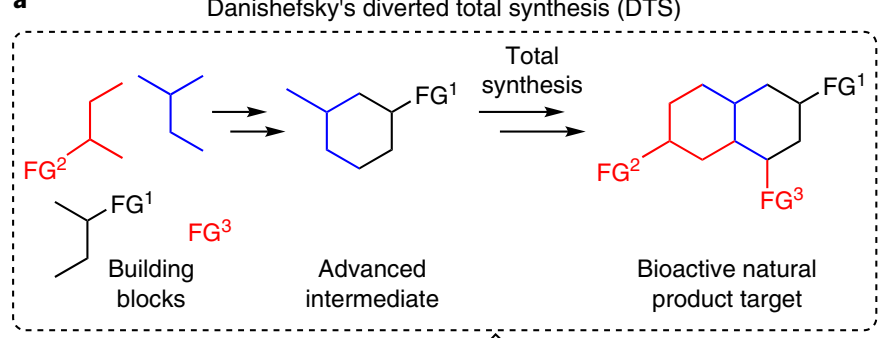

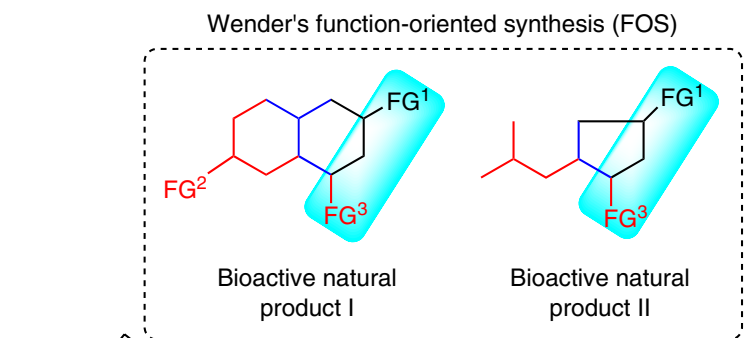

Schreiber's diversity-oriented synthesis (DOS)

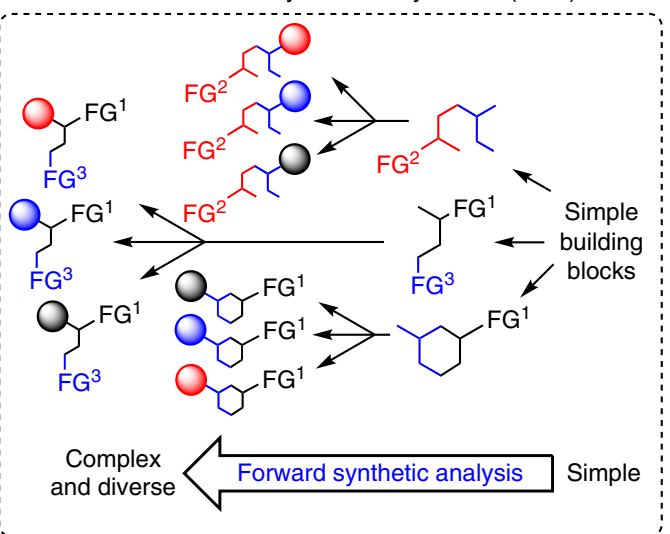

b

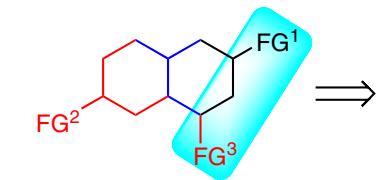

Bioactive natural product target

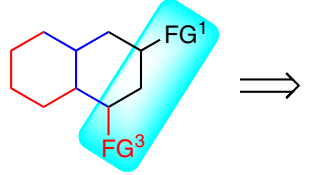

Simplified structures bearing

proposed pharmacophore

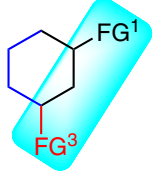

re

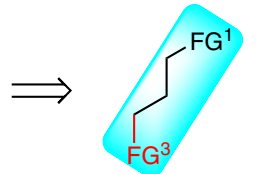

Proposed pharmacophore

\section{And additional pharmacophore hypotheses leading to alternative retrosyntheses}

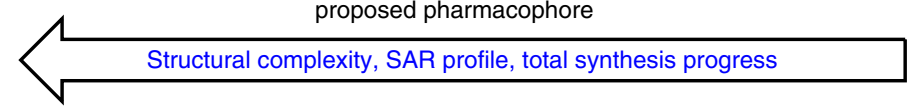

Fig. 1 | Pharmacophore-directed retrosynthesis (PDR) applied to gracilin A and comparison to other synthetic strategies harvesting the rich information content of natural products. a, Strategies toward simplified, bioactive small molecules using natural products as starting points, including diversityoriented synthesis (DOS) directed towards libraries of natural product-inspired compounds. b. PDR seeks to identify or hypothesize the pharmacophore of a natural product, dictating retrosynthesis by ensuring these features are present in multiple intermediates towards the natural product target.

analysis with chemical intuition; (2) existing SAR from isolated natural product congeners; (3) the activity of structurally related compounds; (4) anticipated reactivity. A retrosynthesis is then devised that ensures that the proposed pharmacophore is present in multiple intermediates of increasing complexity, ultimately leading to the natural product. We selected a member of the spongiane diterpenoid family, gracilin A $(\mathbf{1})^{18}$, to initiate assessment of the utility of PDR toward exploring its recently described immunosuppressive ${ }^{19}$ and neuroprotective activity ${ }^{20}$ given the limited SAR information and no prior synthetic work (Fig. 2a) ${ }^{21}$. In the retrosynthetic analysis, as complexity is increased towards the natural product, several intermediates possessing the proposed pharmacophore are specifically targeted, thus enabling SAR to be gathered as the total synthesis progresses (for example, $8 \rightarrow \mathbf{7} \rightarrow \mathbf{6} \rightarrow \mathbf{1}$ in Fig. $2 \mathrm{~b}$ ). It should be noted that several hypotheses regarding the pharmacophore of a particular natural product could be posited for PDR, leading to alternative retrosynthetic strategies.

The gracilins, including the rare nor-diterpene gracilin $\mathrm{A}^{18}$, were originally isolated and characterized from the Mediterranean sponge Spongionella gracilis $^{22}$. These diterpenes are structurally unique owing to the unusual diacetoxy furanose found in most members. The cytotoxic activity of gracilins B and G-I (isolated from Spongionella pulchella) against a diverse panel of 12 human cancer cell lines has been reported, but these compounds did not progress further in preclinical evaluation ${ }^{23}$. The absolute configuration of gracilin A was never established, while its relative configuration was based on X-ray analysis of the keto derivative of 9,11-dihydrogracilin A (3) ${ }^{24}$. Gracilin A was reported to be a potent inhibitor of phospholipase A2 (PLA2) with a 69\% inactivation efficiency $^{25}$. We previously reported that gracilin A was mildly cytotoxic against K562 and peripheral blood mononuclear cells (with half-maximum inhibitory concentration $\left(\mathrm{IC}_{50}\right)$ values of 0.6 and $0.8 \mu \mathrm{M}$, respectively) and inhibited the epidermal growth factor receptor by $70 \%$ at $100 \mu \mathrm{M}$ concentration ${ }^{22}$. We also recently posited that gracilin A mimics the effects of cyclosporin A (CsA) through interaction with cyclophilin A (CypA) ${ }^{19}$ and also improves the hallmarks of Alzheimer's disease in vitro and in vivo ${ }^{20,26,27}$. These results prompted the current study to apply PDR to unravel and ideally differentiate the structural requirements for the immunosuppressive and neuroprotective effects observed with gracilin A.

The cyclophilins (Cyp) are highly conserved peptidyl-prolyl, cis-trans isomerases (PPIase) involved in protein folding and trafficking ${ }^{28-30}$ and are found in multiple cellular compartments ${ }^{31}$. CypA, the cytoplasmic isoform, has several roles in cell metabolism 


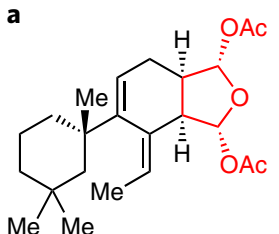

Gracilin A (1)<smiles>C/C=C1\C(C)=C[C@@H]2C1=C[C@H](O)[C@H]1[C@H](OC(C)=O)O[C@H](OC(C)=O)[C@@H]21</smiles>

Gracilin L (2)

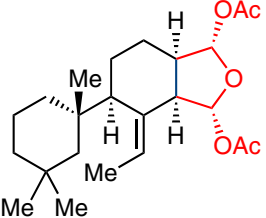

9,11-Dihydrogracilin A (3)<smiles>CC(=O)O[C@H]1O[C@H](OC(C)=O)[C@H]2[C@@H]1CC[C@H](C)[C@]21CCCC(C)(C)C1</smiles>

Dendrillin (4)

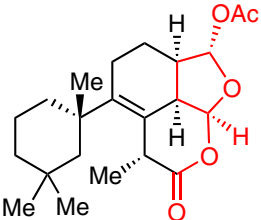

Tetrahydroaplysulphurin-1 (5)
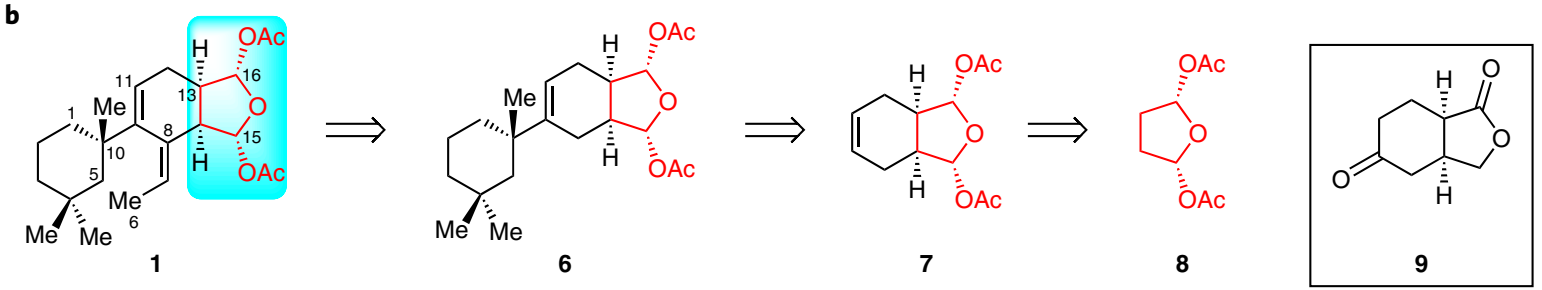

Fig. 2 | Select members of the gracilin A family and application of PDR to gracilin A. a, Naturally occurring spongiane diterpenoids bearing a common bis-acetoxy furanose moiety (red) selected as the hypothetical pharmacophore. $\mathbf{b}$, PDR applied to gracilin A (1) leads to increasingly complex intermediates (that is, $\mathbf{8}, \mathbf{7}, \mathbf{6}$ ) throughout the course of total synthesis from key intermediate $\mathbf{9}$.

and energy homeostasis ${ }^{32}$, with enhanced expression in inflammation and cancer ${ }^{33}$. The use of small molecules that selectively block the inflammation-related functions of CypA, is an important pharmacological strategy leading to effective immunosuppressive agents $^{33}$. On the other hand, cyclophilin D (CypD), the mitochondrial isoform, translocates to form the mitochondrial permeability transition pore (mPTP) and its activity correlates with the mitochondrial dysfunction observed in Alzheimer's disease that leads to neuronal death ${ }^{34}$. CypD inhibitors devoid of immunosuppressive activity through lack of binding to CypA but possessing the desired mitochondrial effect and appropriate blood-brain barrier permeability could provide an approach to address Alzheimer's disease.

Herein, we demonstrate a proof-of-principle study of PDR through application to the spongiane diterpene, gracilin $\mathrm{A}$, that has led to useful lead compounds for both immunosuppression and neuroprotection. This study revealed simplified gracilin A derivatives with high affinity for CypA and others that demonstrated significant selectivity for CypD over CypA, demonstrating their potential as neuroprotective agents devoid of immunosuppressive effects.

\section{Results and discussion}

Pharmacophore-directed retrosynthesis applied to gracilin A. The bis-acetoxy furanose moiety was selected as the pharmacophore of gracilin A based on several lines of evidence. Studies of the reactivity of macfarlandin $\mathrm{E}$ (which possesses a 1,4-dicarbonyl masked as a bis-acyloxy furanose) with lysine derivatives provided evidence for pyrrole formation through a Paal-Knorr process ${ }^{35}$, and mounting evidence suggests that this is possible with a number of other bis-acetoxy furanose-containing natural products ${ }^{36}$. Furthermore, a computational study demonstrated the potential of the bis-acyloxy furanoses of gracilin A and aplysulphurin-1 to bind divalent cations such as $\mathrm{Ca}^{2+}$, pointing to the potential importance of this moiety for bioactivity ${ }^{37}$. Finally, it is interesting to note that several bioactive members of the spongiane diterpenoid family possess a bis-acyloxy furanose including the structurally related gracilin L $(2)^{22}, 9$,11-dihydrogracilin A (3), dendrillin (4) ${ }^{38}$ and tetrahydroaplysulphurin-1 (5) ${ }^{39}$. These considerations guided application of PDR to gracilin A and imposed a requirement that multiple intermediates along the synthetic route would bear or be converted to the proposed pharmacophore, namely the bis-acetoxy furanose (for example, derivatives 6-8, Fig. 2b). In this way, SAR studies could be conducted throughout the course of the total synthesis.
We recognized that keto lactone $\mathbf{9}$, accessible in gram quantities through our recently described Diels-Alder lactonization organocascade $^{40}$, would serve as a key intermediate to study the importance of the C8-exocyclic alkene (for example derivative 6), the C9-appended cyclohexyl moiety (for example, derivative 7). The importance of the bicyclic core of gracilin A would be ascertained by synthesis of the highly simplified monocyclic furanose $\mathbf{8}$ (Fig. 2b).

Sequential synthesis of increasingly complex gracilin A derivatives. We recognized that, in applying PDR, synthetic progress with concurrent biological assays of intermediates could best be achieved in stages based on the increasing complexity of intermediates in the synthetic sequence, as outlined in Figs. $3 \mathrm{a}-\mathrm{c}$ and $4 \mathrm{a}$. A final stage of diverted total synthesis could enable further refinement of the SAR profile through 'gap filling' with particular targeted derivatives to answer more specific questions building on information gathered during the initial stages. As applied to gracilin A, we first targeted the simple bis-acetoxy furanose $\mathbf{8}$ as the minimal pharmacophore that was readily obtained through a two-step oxidation/hydrogenation sequence to deliver the racemic syn-substituted acetoxy furanose $( \pm)-8 \mathbf{a}$ along with the anti-diastereomer $( \pm)$-8b (d.r. 1.7:1). In a second stage, derivatives devoid of the cyclohexyl substituent and exocyclic alkene were targeted, namely bicyclic bis-acetoxy furanose 7a,b (Fig. 3b). The bicyclic lactone endo-15a (3:1 d.r., 94\% e.e.) was obtained from a Diels-Alder-lactonization organocascade employing diene 12 and acryloyl chloride (11) with tetramisole as the Lewis base promoter. The required endo-diastereomer 15 a could be isolated in $58 \%$ yield, and subsequent reduction with $\mathrm{LiAlH}_{4}$ followed by desilylation delivered ketodiol 16. Swern oxidation led to a dialdehyde that was directly subjected to acid-promoted acetylation to give the unstable keto bis-acetoxy furanoses $\mathbf{1 7}$ as a mixture of syn/anti diastereomers. A subsequent reduction with $\mathrm{NaBH}_{4}$ and dehydration with $\mathrm{SOCl}_{2}$ delivered the bicyclic bis-acetoxy furanoses $\mathbf{7 a}, \mathbf{b}$ as a mixture of alkene regioisomers. The low yields obtained in this and subsequent four-step sequences leading to the bis-acetoxy furanoses were primarily a result of incomplete or non-simultaneous Swern oxidation of the diols leading to regioisomeric monoacetoxy furanoses.

We next targeted derivatives devoid of the exocyclic alkene but bearing the cyclohexyl substituent (Fig. 3c). Desilylation of endo-Diels-Alder adduct 15a gave bicyclic lactone (-)-9, which was subjected to an allylzinc reagent derived from cyclohexenyl choride $( \pm)$-18 employing conditions we previously used in our 
a<smiles>c1ccoc1</smiles>

10
(1) $\mathrm{Pb}(\mathrm{OAc})_{4}, \mathrm{AcOH}, 23{ }^{\circ} \mathrm{C}, 20 \mathrm{~h}$

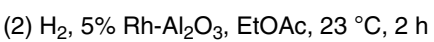
(94\%, 2 steps, 1.7:1 syn/anti)<smiles>C=CC(=O)Cl</smiles>

14<smiles>COC(=O)OC1CCC(OC(=O)OC2CCOC2=O)O1</smiles>

$( \pm)-8 b$

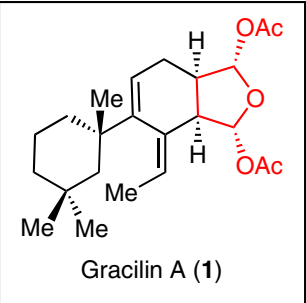

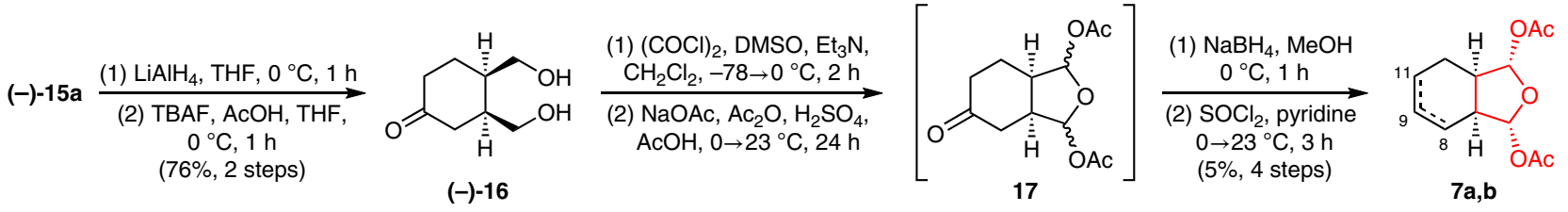

c

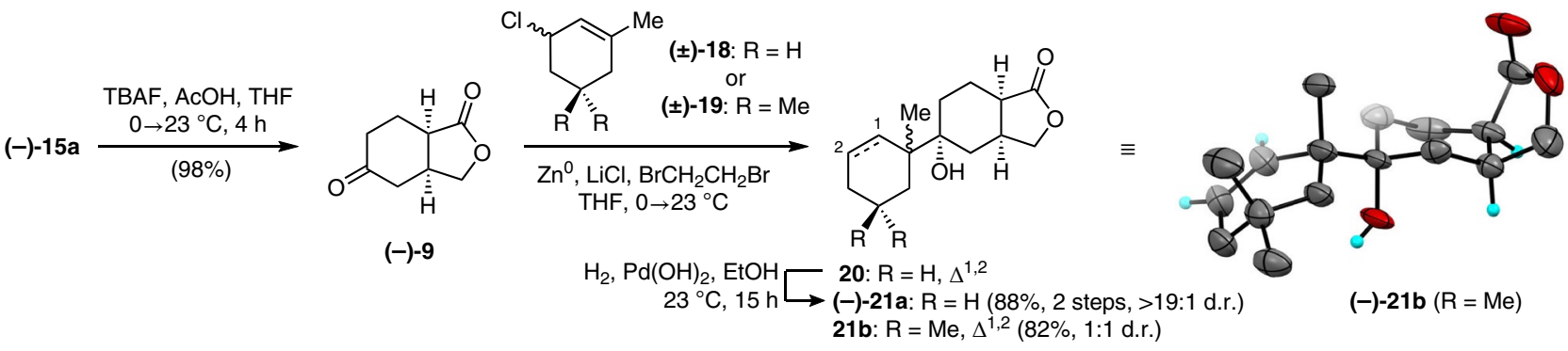

(1) $\mathrm{H}_{2}, \mathrm{Pd}(\mathrm{OH})_{2}, \mathrm{EtOH}, 23^{\circ} \mathrm{C}, 15 \mathrm{~h}$

(2) $\mathrm{LiAlH}_{4}, \mathrm{THF}, 0^{\circ} \mathrm{C}, 1 \mathrm{~h}$

(3) $(\mathrm{COCl})_{2}, \mathrm{DMSO}, \mathrm{Et}_{3} \mathrm{~N}$

$\mathrm{CH}_{2} \mathrm{Cl}_{2},-78 \rightarrow 23^{\circ} \mathrm{C}, 3 \mathrm{~h}$

21b
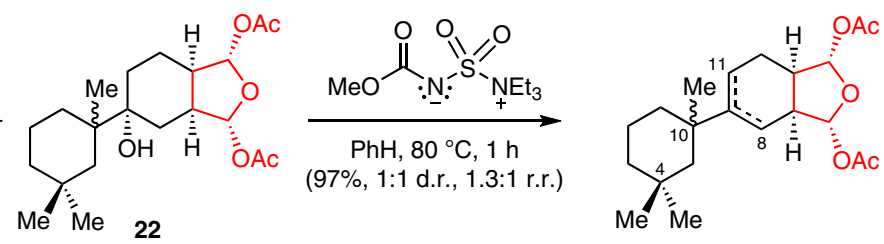

$\mathrm{AcOH}, 0 \rightarrow 23^{\circ} \mathrm{C}, 48 \mathrm{~h}$

(37\%, 4 steps, $1: 1$ d.r.)

22

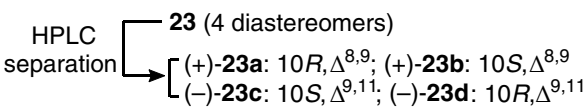

Fig. 3 | Pharmacophore-directed retrosynthesis applied to gracilin A. a, Synthesis of a highly simplified analogue bearing the proposed pharmacophore. b. Assembly of the cis-fused, 6,5-bicyclic core 15a of gracilin A through an enantioselective, organocatalytic Diels-Alder-lactonization cascade and manipulation to a simplified bicyclic analogue 7. c, Annulation of the natural and a simplified cyclohexyl moiety. Inset, ORTEP representation of the single crystal X-ray structure of (-)-21b. TBS, tert-butyldimethyl silyl; r.r., regioisomeric ratio.

synthesis of spongiolactone $\mathrm{e}^{41}$. This delivered the tricyclic adducts 20 as a 1:1 mixture of diastereomers at the generated quaternary carbon but with high facial selectivity, leading to a single epimer at the tertiary alcohol centre. The contra-steric addition of the allylzinc reagent to the concave face of ketone (-)-9 was unexpected but likely due to stereoelectronic effects leading directly to a chair rather than a boat conformation and was verified by extensive NMR studies (see Supplementary Section C for details). In the case of addition of the gem-dimethyl cyclohexenyl zinc reagent derived from $( \pm)-19$, the stereochemistry was further verified by single-crystal $\mathrm{X}$-ray analysis of adduct (-)-21b. However, this diastereoselectivity is inconsequential because the tertiary alcohol in (-)-21b is subsequently dehydrated. Hydrogenation reduced the cyclohexene of (-)-21b and a three-step process delivered the hydroxy bisacetoxy furanose 22 as a 1:1 mixture of diastereomers. Dehydration with Burgess' reagent ${ }^{42}$ gave alkenes 23 as a mixture of four diastereomers due to the alkene regioisomers produced. The four diastereomers were separable by preparative chiral HPLC, enabling biological analysis of each stereoisomer.

Gaps in the SAR profile were also back-filled following initial assays as described in the following (Fig. 4). This entailed application of aspects of diverted total synthesis to synthesize derivatives devoid of the cyclohexyl moiety (that is, 24), variations in the oxidation state and substituents of the tetrahydrofuran and cyclohexyl 
$(-)-15 a$

$$
\begin{gathered}
\text { (1) DIBAl-H, } \mathrm{CH}_{2} \mathrm{Cl}_{2},-78{ }^{\circ} \mathrm{C} .3 \mathrm{~h} \\
\text { (2) } \mathrm{TBAF}, \mathrm{AcOH}, \mathrm{THF} \\
0 \rightarrow 23^{\circ} \mathrm{C}, 4 \mathrm{~h} \\
(81 \%, 2 \text { steps, } 4: 1 \text { d.r. }) \\
\text { (3) } \mathrm{Ac}_{2} \mathrm{O}, \text { DMAP, pyridine, } 23^{\circ} \mathrm{C}, 4 \mathrm{~h}
\end{gathered}
$$

$(98 \%,>19: 1$ d.r. $)$

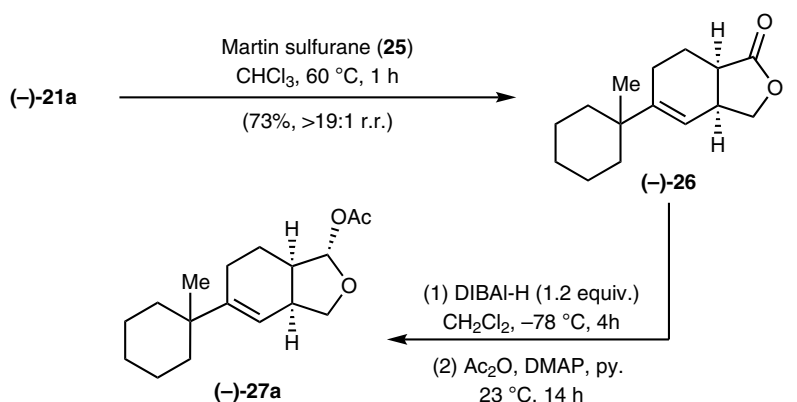

$(60 \%, 2$ steps, $>19: 1$ d.r. $)$
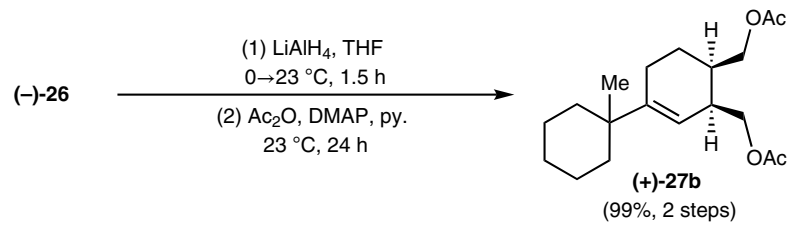

$(+)-15 a$
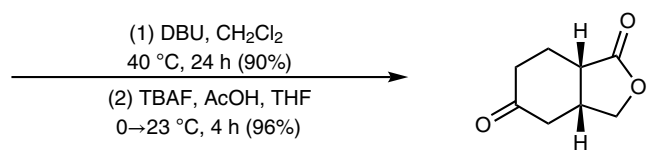

$(+)-9$

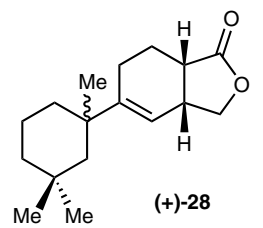

(1) (土)-19, $\mathrm{Zn}^{0}, \mathrm{LiCl}, \mathrm{BrCH}_{2} \mathrm{CH}_{2} \mathrm{Br}$ $\mathrm{THF}, 0 \rightarrow 23^{\circ} \mathrm{C}, 14 \mathrm{~h}$ $(78 \%, 1: 1$ d.r. $)$

(2) $\mathrm{H}_{2}, \mathrm{Pd}(\mathrm{OH})_{2}, \mathrm{EtOH}, 23^{\circ} \mathrm{C}, 15 \mathrm{~h}$

(3) Martin sulfurane, $\mathrm{CHCl}_{3}, 60^{\circ} \mathrm{C}, 1 \mathrm{~h}$

(79\%, 2 steps, $1: 1$ d.r., $>19: 1$ r.r.)

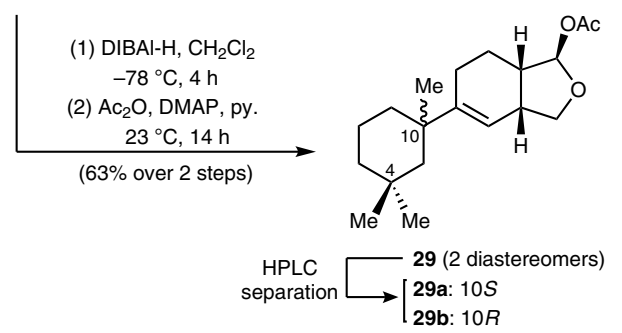

Fig. 4 | Synthesis of gracilin A derivatives toward SAR profile gap filling. a, Alternative oxidation states of the original bis-acetoxy furanose. $\mathbf{b}$, Select gracilin A derivatives in the enantiomeric series.

moiety (that is, 26-27), and also exploration of the enantiomeric series (that is, 28-29).

PDR enables sequential increases in complexity with correlation to immunosuppressive activity. As a preliminary biological screen, the initial gracilin A derivatives synthesized were analysed by surface plasmon resonance (SPR) with immobilized $\mathrm{CypA}^{43,44}$. Biological testing of gracilin A derivatives was performed in sets as the various stages of PDR were accomplished (Figs. $3 \mathrm{a}-\mathrm{c}$ and $4 \mathrm{a}, \mathrm{b}$ ). Association curves $\left(K_{\mathrm{D}}\right)$ were measured at various concentrations in comparison to gracilin A and CsA and the initially synthesized highly simplified derivatives, monocyclic ( \pm )-8 and bicyclic 7 bis-acetoxy furanoses, exhibited no affinity for CypA up to $10 \mu \mathrm{M}$ (Fig. 5). On binding to CypA, CsA and gracilin A modulate interleukin 2 (IL-2) release through the calcineurin pathway ${ }^{19}$, so the effects of these simplified derivatives on IL-2 production were also measured. Concanavalin A (ConA) was used to activate human $\mathrm{T}$ lymphocytes and induce IL-2 release ${ }^{45}$. T lymphocytes were pre-treated for $2 \mathrm{~h}$ with different non-toxic concentrations of compounds and then activated for $48 \mathrm{~h}$ in the presence of ConA $\left(50 \mu \mathrm{g} \mathrm{ml}^{-1}\right)$. After this time, levels of IL-2 released to the medium were measured by an enzyme-linked immunosorbent assay (ELISA) kit. As expected, the greatly simplified monocyclic $( \pm)-\mathbf{8 a}, \mathbf{b}$ and bicyclic furanoses $\mathbf{7 a}, \mathbf{b}$ did not inhibit IL-2 production nor exhibit T-lymphocyte toxicity at concentrations up to $10 \mu \mathrm{M}$ (MTT assay).

Gracilin A derivative $\mathbf{2 3}$ bearing the pendant cyclohexyl moiety, began to display immunosuppressive activity (Fig. 5). The initial diastereomeric mixture of tertiary alcohols $\mathbf{2 2}$ derived from cyclohexenyl zinc addition was inactive. However, following dehydration to introduce the tri-substituted alkenes, immunosuppressive activity was observed with only a twofold decrease in $K_{\mathrm{D}}$ relative to gracilin A $(2.53 \pm 0.40 \mu \mathrm{M})$ for alkene $23 \mathrm{~b}$ bearing the $10 S$ configuration $(5.83 \pm 3.33 \mu \mathrm{M})$ with moderate IL-2 inhibition $\left(\mathrm{IC}_{50} 0.30 \mu \mathrm{M}\right)$. The interplay between alkene regioisomers $\left(\Delta^{8,9}\right.$ versus $\left.\Delta^{9,11}\right)$ and the C10 configuration was revealed through derivatives 23a and 23d, which exhibited nearly a 1,000 -fold increase in $K_{\mathrm{D}}$ toward CypA compared to gracilin A $(5.34 \pm 1.68 \mathrm{nM}$ and $7.57 \pm 1.61 \mathrm{nM}$, respectively), while isomers $\mathbf{2 3 b}$ and $\mathbf{2 3 c}$ were either significantly less active or completely inactive, respectively. Derivatives 29a and 23a displayed $\mathrm{IC}_{50}$ values for IL-2 inhibition of 0.12 and $0.15 \mu \mathrm{M}$, respectively whereas the $\mathrm{C} 10$ epimer of $\mathbf{2 3 a}$, derivative $\mathbf{2 3 b}$ and the $\Delta^{9,11}$ regioisomer 23d, had higher values of 0.30 and $0.36 \mu \mathrm{M}$, respectively, while the epimeric, $\Delta^{9,11}$ regioisomer $23 \mathrm{c}$ exhibited a value of $>10 \mu \mathrm{M}$, again pointing to the interplay between the alkene regiochemistry and the C10 stereochemistry for IL-2 inhibition. The discrepancy between binding $\left(K_{\mathrm{D}}\right)$ to CypA as measured by SPR and the cellular inhibition of IL-2 expression may point to differential cell permeability or reflect differential binding to calcineurin, the presumed target of the CypA-gracilin A complex, impacted by the absence of the $\mathrm{C} 8$ exocyclic ethylidene. In addition, the conformation of the cyclophilins is highly dependent on the $\mathrm{pH}$ of the assay. This assay was performed at low $\mathrm{pH}$ (4.5) to demonstrate the activity of gracilin derivatives, which also lowers the $K_{\mathrm{D}}$ for CsA compared to literature values (see Supplementary Section B and Discussion for details). To address this discrepancy, we also performed an enzymatic assay to measure calcineurin inhibition by gracilin $\mathrm{A}$ derivatives (at $1 \mu \mathrm{M})$ and, as expected, the most potent derivatives in the SPR assay showed comparable inhibition (22-31\%) to CsA $(23 \%$ at $1 \mu \mathrm{M})$. Despite differences in CypA binding affinity, these derivatives have comparable activity in both IL-2 and calcineurin assays, which supports the hypothesis that these derivatives lead to differential binding to calcineurin by the respective CypA-gracilin derivative complexes. These data, taken together, suggest that conformational preferences about the $\mathrm{C} 9-\mathrm{C} 10$ bond play a pivotal role in the immunosuppressive activity of the gracilin family.

To fill gaps in the SAR profile regarding immunosuppressive activity, we turned to the application of diverted total synthesis and targeted additional simplified derivatives (Fig. 5). The greatly simplified bicyclic mono-acetoxy ketone $\mathbf{2 4}$ and the bicyclic lactone $\mathbf{2 8}$, differing from the highly active bis-acetoxy furanose 23a only by the oxidation state of the tetrahydrofuran, were completely inactive. Epimerization of exo-15b enabled access to the enantiomeric series, leading to mono-acetoxy furanoses $29 \mathbf{a}, \mathbf{b}$ epimeric at the quaternary C10 centre. Affinity to CypA $\left(K_{\mathrm{D}} \approx 3 \mu \mathrm{M}\right)$ for both diastereomers 29a,b was comparable to gracilin A; however, differential inhibition of IL- 2 production $\left(\mathrm{IC}_{50}=0.12\right.$ versus $\left.>10 \mu \mathrm{M}\right)$ was observed for both diastereomers while remaining relatively nontoxic to $\mathrm{T}$ cells $\left(\mathrm{EC}_{50}=1-4 \mu \mathrm{M}\right)$. The importance of the quaternary carbon stereochemistry and alkene regisomer on IL-2 inhibition, which directly impacts the conformation about the C9-C10 bond, 


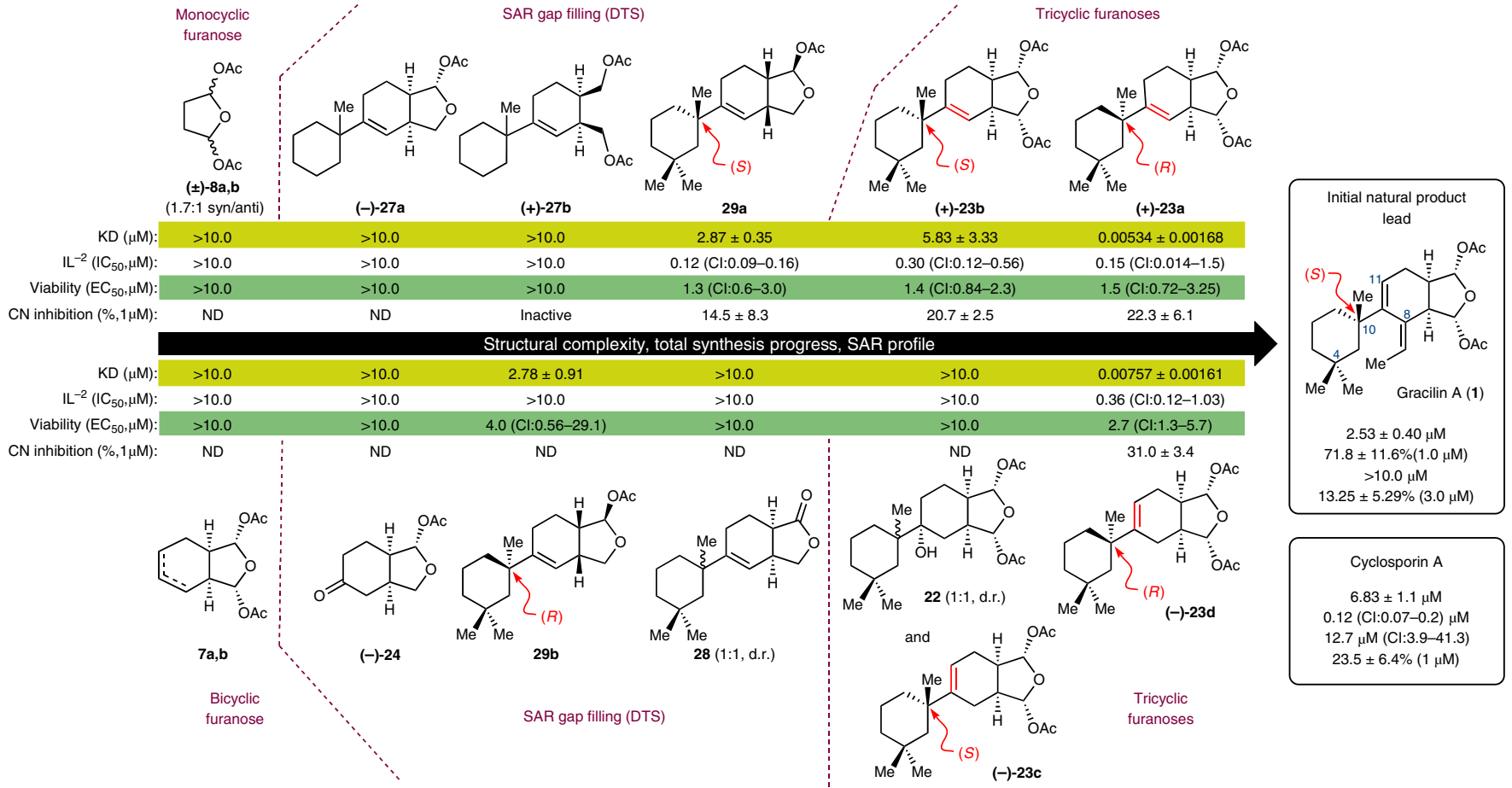

Fig. 5 | Immunosuppressive activity of gracilin A derivatives. Kinetic equilibrium dissociation constants $\left(K_{D}, \mu M\right)$ for binding of gracilin $A$ derivatives to CypA as measured by SPR ( $n=4$, data presented as mean \pm s.e.m.), IL-2 release inhibition $\left(I C_{50}, n=10\right.$, mean, CI) by ELISA, and cell viability of human T lymphocytes (half-maximal inhibitory concentration, $\mathrm{EC}_{50}, n=3$, mean, $\mathrm{Cl}$ ) as determined by an $\mathrm{MTT}$ assay. $\mathrm{Cl}$ ( $95 \%$ confidence interval); $R^{2}$ values (0.91-0.98) were in the acceptable range (see Supplementary Section B for details). Inhibition of calcineurin (CN) phosphatase activity at $1 \mu M$ expressed in percentage of control cells $(n=4$, mean \pm s.e.m. $)$. $(>10.0=$ not active up to $10 \mu \mathrm{M})$. ND, not determined.

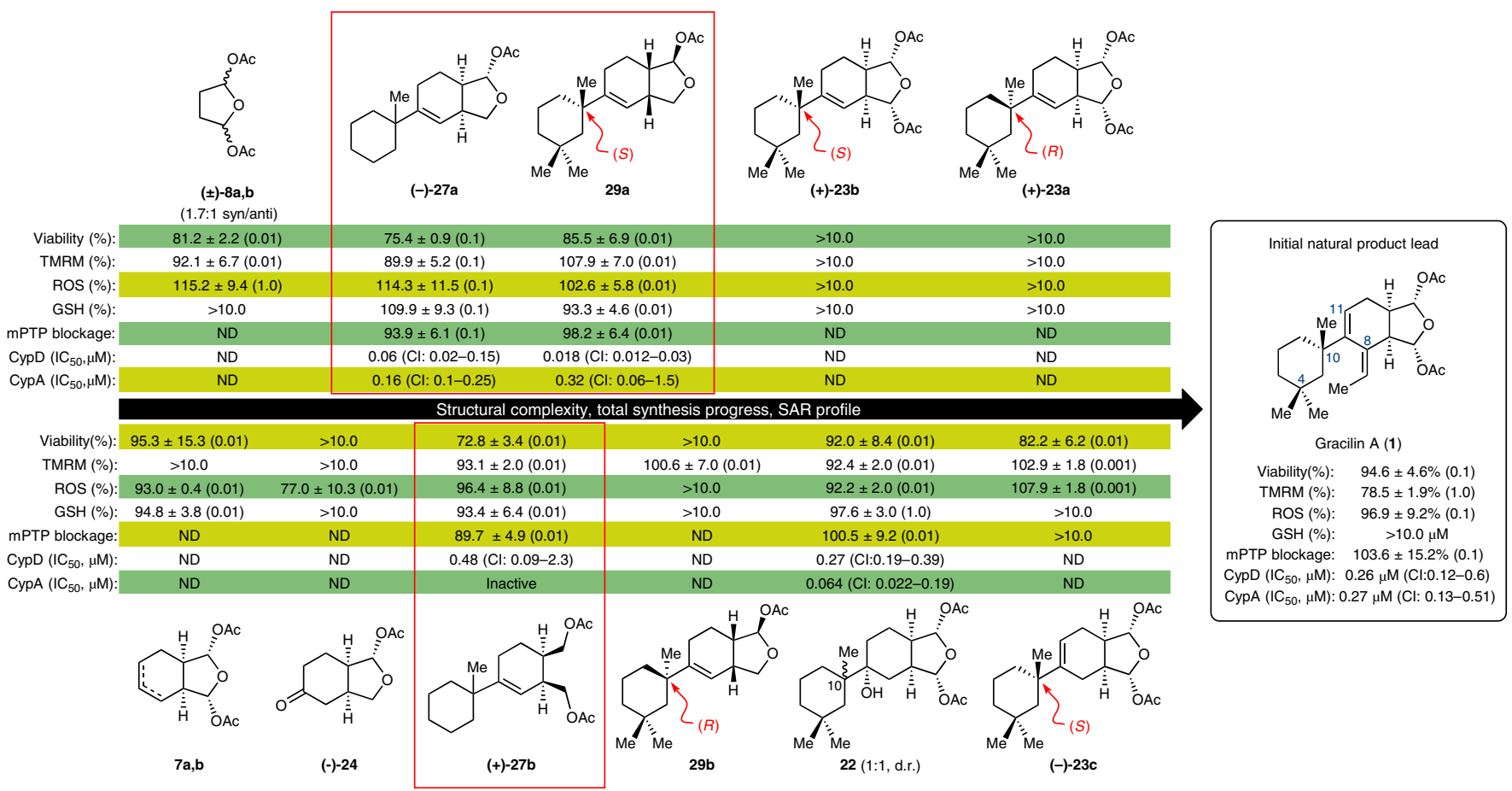

Fig. 6 | Activity of gracilin A derivatives as neuroprotective agents. Cell viability against $\mathrm{H}_{2} \mathrm{O}_{2}$ toxicity, mitochondrial membrane potential restoration

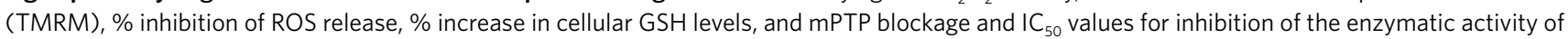
CypD and CypA. The lowest effective concentration of each derivative is indicated in parentheses $(\mu \mathrm{M})$. Values are calculated as the percentage of untreated control cells in comparison to $\mathrm{H}_{2} \mathrm{O}_{2}$-treated cells with the following values: $\mathrm{H}_{2} \mathrm{O}_{2}$ toxicity $55.6 \pm 4.4, T M R M 75.9 \pm 3, \mathrm{ROS} 140.3 \pm 4.4, \mathrm{GSH}$ $82.9 \pm 1.6$, mPTP blockage $76.7 \pm 2.8\left(n=4\right.$, mean \pm s.e.m.). Cyp D and A activity are expressed as $I C_{50}$ values $(\mu \mathrm{M})$. Cl ( $95 \%$ confidence interval); $R^{2}$ values (0.91-0.99) were in the acceptable range (see Supplementary Section C for details); derivatives exhibiting neuroprotective effects while also showing differential activity of CypA versus CypD are highlighted (red boxes; $n=4$, mean \pm s.e.m.) (>10.0 = not active up to $10 \mu M$ ). ND, not determined. 
is again highlighted by this enantiomeric series with the natural $(S)$ configuration imparting the greatest activity.

Neuroprotective activity of gracilin A derivatives. Given the neuroprotective effects previously observed for gracilin $\mathrm{A}$, the derivatives synthesized through application of PDR for immunosuppressive effects were also studied for potential neuroprotective activity and of particular interest was possible selectivity for CypD versus CypA PPiase activity (Fig. 6), a key property required for neuroprotective lead compounds devoid of immunosuppressive effects. All assays were performed at the indicated concentrations $(0.001,0.01,0.1$ or $1.0 \mu \mathrm{M})$ based on cytotoxicity determined with SH-SY5Y cells. In particular, compounds that displayed some toxicity against this cell line at $1 \mu \mathrm{M}$ were tested at lower concentrations. Treatment of SH-SY5Y cells with a known potent oxidant such as $\mathrm{H}_{2} \mathrm{O}_{2}$ produces oxidative damage with a consequent increase in reactive oxygen species (ROS) release. ROS generation provokes mitochondrial dysfunction, increases cell death and affects the cellular antioxidant systems such as the glutathione (GSH) cycle ${ }^{46}$. Therefore, four parameters were measured to evaluate the neuroprotective effects of the simplified gracilin A derivatives: cell viability, mitochondrial membrane potential $\left(\Delta \Psi_{\mathrm{m}}\right)$, ROS release and GSH levels after cellular challenge with $\mathrm{H}_{2} \mathrm{O}_{2}$ (see Supplementary Section $\mathrm{C}$ for details). Several highly simplified gracilin A derivatives, namely monoacetoxy furanose $\mathbf{2 7 a}$ and the highly simplified diacetate 27b (both derived from 21a), the inseparable, tertiary alcohols 22 (1:1, d.r.) and the enantiomeric mono-acetoxy furanose 29a, but not its diastereomer 29b, and even the simplest bisacetoxy furanose $( \pm)-\mathbf{8 a}, \mathbf{b}(1.7: 1$, syn/anti) displayed significant neuroprotective effects. In view of these results, the compounds with greater activity in oxidative stress assays $(\mathbf{2 7 a}, \mathbf{2 7} \mathbf{b}, \mathbf{2 9 a}, 22$ and $23 \mathrm{c}$ ) were chosen to test their ability to block the opening of the mPTP. The simplest derivatives $\mathbf{8 a}, \mathbf{b}$ and $\mathbf{7 a}, \mathbf{b}$ displayed lower activities and only at one of the concentrations tested, so these compounds were not subjected to the following assays. In PPIase activity assays, selectivity for CypD over CypA was observed for derivatives 27a ( 3-fold) and 29a ( $\sim 18$-fold) with the very simple diacetate 27b displaying the greatest differential CypD activity because it was inactive against CypA (up to $10 \mu \mathrm{M}$ ) but displayed activity against $\mathrm{CypD}\left(\mathrm{IC}_{50}=0.48 \mu \mathrm{M}\right)$. In contrast, tertiary alcohols 22 had opposite selectivity for CypA over CypD ( 4-fold). Our results suggest that the antioxidant effect of these gracilin $\mathrm{A}$ derivatives is mitochondrial-related, similar to gracilin A, mediated through interaction with CypD. These compounds protect cells from oxidative damage induced by $\mathrm{H}_{2} \mathrm{O}_{2}$ improving mitochondrial functioning measured by MTT and TMRM assays and increased GSH levels. Moreover, they block mitochondrial pore opening and induce CypD activity inhibition. Therefore, gracilin A derivatives hold potential as neuroprotective lead compounds that are devoid of immunosuppressive effects.

Summary of PDR and SARs gleaned from application of PDR to gracilin A. The total synthesis of natural products continues to be an important endeavour for the discovery of novel synthetic strategies and methods in addition to the exploration of biologically relevant chemical space. The described PDR approach brings biological function into the retrosynthetic planning stages to target multiple, simplified derivatives bearing a hypothesized pharmacophore en route to the natural product. PDR can be considered to be a subset of Wender's function-oriented synthesis and will of course not be applicable to every natural product (that is, those where the majority of the structure is required for bioactivity). A caveat to this approach is that a balance must be found between synthetic convergency and targeting of bioactivity during the total synthesis. However, the application of PDR may only be limited by the creativity and intuition of synthetic chemists building on minimal SAR.

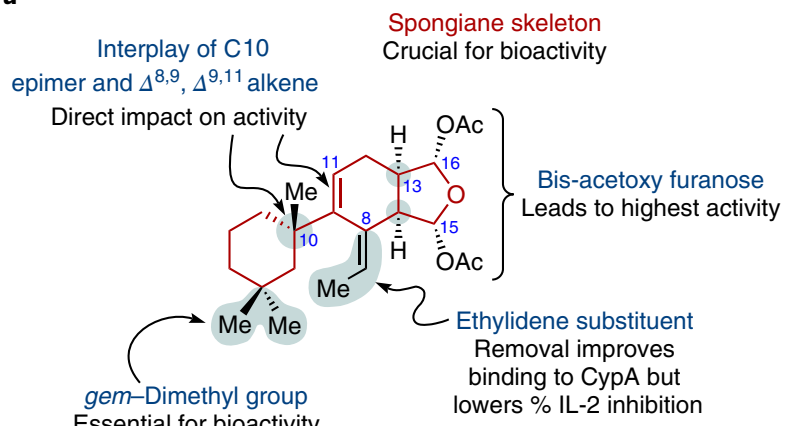

b

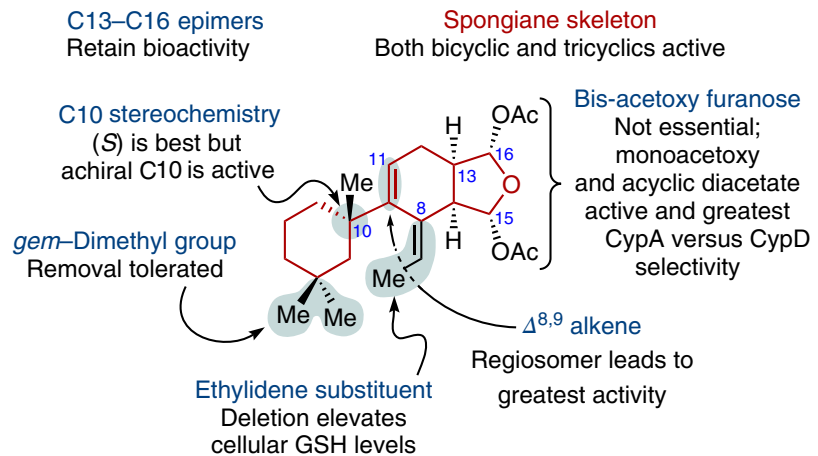

Fig. 7 | SAR profile of gracilin A for both immunosuppressive and neuroprotective activity enabled through application of PDR. $\mathbf{a}$, SAR profile in immunosuppressive assays. b, SAR profile in neuroprotective assays.

For example, the SARs of isolated natural product congeners and other lines of evidence may direct one or more hypotheses regarding a proposed pharmacophore. Indeed, application of PDR can generate alternative strategies for a given natural product through alternative hypothesized pharmacophores in a similar way to consideration of various strategic bond disconnections in classic retrosynthesis. In the present study, the first SAR profiles of gracilin A derivatives were secured by providing proof-of-principle studies of PDR and support the notion of the bis-acetoxy furanose as a pharmacophore for immunosuppressive activity but not necessarily for neuroprotective activity (Fig. 7).

Evidence was gathered for our initial hypothesis invoking the bis-acetoxy furanose of gracilin $\mathrm{A}$ as the pharmacophore, as relates to immunosuppressive activity, given that derivatives with this moiety (for example, 23a and 23d) displayed the greatest activity $\left(K_{\mathrm{D}} \approx 5-7 \mathrm{nM}\right)$, while those lacking this moiety were inactive (for example, (-)-28). Importantly, PDR disclosed that the C8-ethylidene is not required to elicit potent immunosuppressive activity and, with the availability of natural gracilin A as a comparator, it was unnecessary to complete a total synthesis.

While derivatives of CsA were previously investigated as potential neuroprotective agents through inhibition of CypD, they lacked effectiveness due to their high molecular weights and low blood-brain barrier permeability ${ }^{47-49}$. Several gracilin A derivatives (for example, 27a,b and 29a) accessed through this study, that interestingly includes the enantiomeric series, displayed significant neuroprotective activity, importantly while also displaying selectivity for CypD versus CypA inhibition. These gracilin derivatives serve as lead compounds for neurodegenerative diseases and other CypD-mediated diseases including atherosclerosis or autoimmune diseases ${ }^{50,51}$. 
Although an ideal application of PDR will generally be challenging to implement, in particular when reactive functionality precludes a completely linear synthetic strategy as dictated by PDR, as in the present case of the gracilins, we expect that pharmacophore hypotheses brought into retrosynthetic planning will enable greater SAR information to be gathered en route to a natural product. It is also anticipated that application of PDR will provide an avenue for hypothesis-driven, natural product total synthesis efforts while simultaneously accelerating the exploration of natural product chemical space through total synthesis efforts premised on PDR.

\section{Methods}

Gram-scale Diels-Alder-lactonization organocascade providing $\gamma$-lactones $(-)-15 a$ and $(+)-15 b$, the bicyclic core of gracilin A derivatives. To an ovendried, $250 \mathrm{ml}$ round-bottomed flask equipped with a magnetic stir bar was added silyloxydiene alcohol $12^{52}$ (2.14 g, $10.0 \mathrm{mmol}, 1.0$ equiv), (S)-(-)-TM. $\mathrm{HCl}(2.40 \mathrm{~g}$, $10.0 \mathrm{mmol}, 1.0$ equiv.), 2,6-lutidine $(3.5 \mathrm{ml}, 30.0 \mathrm{mmol}, 3.0$ equiv. $)$ and anhydrous $\mathrm{CH}_{2} \mathrm{Cl}_{2}(100 \mathrm{ml}$, to make a final concentration of silyloxydiene alcohol of $0.1 \mathrm{M})$ at ambient temperature $\left(23^{\circ} \mathrm{C}\right)$. With vigorous stirring, $11(1.05 \mathrm{ml}, 13.0 \mathrm{mmol}$, 1.3 equiv.) in $\mathrm{CH}_{2} \mathrm{Cl}_{2}(9.0 \mathrm{ml})$ was added over a period of $5 \mathrm{~h}$ by syringe pump addition. After stirring for an additional $13 \mathrm{~h}$, the reaction mixture was filtered through a short pad of $\mathrm{SiO}_{2}$ and the filtrate was concentrated by rotary evaporation. Purification by automated flash chromatography $(5 \rightarrow 50 \%$ EtOAc/hexanes) afforded bicyclic $\gamma$-lactones (-)-15a (1.55 g, 58\% yield, $94 \%$ e.e.) and (+)-15b ( $0.51 \mathrm{~g}, 19 \%$ yield, $94 \%$ e.e.). See Supplementary information section A for full characterization of $\mathbf{1 5 a}$ and $\mathbf{1 5 b}$.

Surface activation, ligand immobilization and binding. A Biacore X SPR biosensor with Control Software and BIAevaluation software version 3.0 from Biacore (GE Healthcare) was used to check the binding between gracilin A derivatives and CypA. Sensor surface activation and ligand immobilization were performed by using Hank's balance solution surfactant P20 (HBS-EP) as running buffer at a flow rate of $5 \mu \mathrm{lmin}^{-1}$ and $25^{\circ} \mathrm{C}$. CM5 sensor chips were used as the surface where Cyp A was immobilized as the ligand. The CM5 chip was a glass slide coated with a thin layer of gold with a matrix of carboxymethylated dextran covalently attached. The CM5 chip was activated using an amine coupling kit. Following the manufacturer's instructions, a mixture (1:1 vol/ vol) of 1-ethyl-3- (3-dimetylaminopropyl) carbodiimide hydrochloride (EDC) and $\mathrm{N}$-hydroxysuccinimide (NHS) was applied for $2 \mathrm{~min}$ over the sensor chip. After activation, the ligand, $100 \mathrm{\mu g} \mathrm{ml}^{-1}$ of active human CypA protein dissolved in sodium acetate $10 \mathrm{mM}$ at $\mathrm{pH} 4.5$, was added to be immobilized over a CM5 sensor chip. Finally, ethanolamine- $\mathrm{HCl}$ was injected to deactivate the remaining active esters and to avoid non-specific binding. Analytes (CsA as positive control or synthetic compounds) were added to evaluate the binding with CypA. Once analytes were tested and interaction was observed, individual binding curves were analysed by determining the kinetic constants of the analytes-CypA binding, namely, the observed rate constant $\left(K_{\mathrm{obs}}\right)$, the association rate constant $\left(K_{\mathrm{ass}}\right)$, the dissociation rate constant $\left(K_{\text {diss }}\right)$ and the kinetic equilibrium dissociation constant $\left(K_{\mathrm{D}}\right)$. At equilibrium, by definition, $K_{\text {diss }} / K_{\text {ass }}=K_{\mathrm{D}}$. The pseudo-firstorder association rate constants $K_{\mathrm{obs}}\left(\mathrm{s}^{-1}\right)$ were determined for each compound concentration by using the 1:1 Langmuir association model of BiaEvaluation software (BiaCore). A representation of $K_{\text {obs }}$ against the corresponding concentration of each compound was then carried out. These plots follow a linear correlation coefficient. From the equation of these representations, $K_{\text {ass }}\left(\mathrm{M}^{-1} \mathrm{~s}^{-1}\right)$, the gradient of the plot, and $K_{\text {diss }}\left(\mathrm{s}^{-1}\right)$, the intercept of the plot, were obtained. From these two values, the kinetic equilibrium dissociation constant $K_{\mathrm{D}}$ for each analyte-CypA binding was obtained.

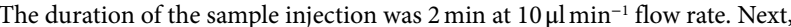
dissociation of bound molecules in HBS-EP buffer flow was studied. The bound drugs were removed from the chip surface before the next injection by adding $1 \mathrm{M}$ glycine- $\mathrm{HCl}$ at $\mathrm{pH} 2.5$ for $1 \mathrm{~min}$. The association phase was used to quantify the compound-CypA interactions. All experiments were performed four times.

Determination of intracellular ROS levels. Intracellular levels of ROS were determined with carboxy- $\mathrm{H}_{2}$ DCFDA (5-(and-6)-carboxy- $2^{\prime}, 7^{\prime}$ dichlorodihydrofluorescein diacetate). This dye diffuses through the cellular membrane and is converted by cellular esterases to carboxy- $\mathrm{H}_{2} \mathrm{DCFH}$ (nonfluorescent). When carboxy- $\mathrm{H}_{2} \mathrm{DCFH}$ is oxidized by ROS, it becomes fluorescent ${ }^{53}$ Cells were seeded at a density of $2.5 \times 10^{5} \mathrm{cells} \mathrm{ml}^{-1}$ in 96 -well plates and allowed to attach for $24 \mathrm{~h}$. Following treatment with compounds at various concentrations $(1 \mu \mathrm{M} \rightarrow 1 \mathrm{nM})$ and $150 \mu \mathrm{M} \mathrm{H}_{2} \mathrm{O}_{2}$ for $6 \mathrm{~h}, \mathrm{SH}-\mathrm{SY} 5 \mathrm{Y}$ cells were washed twice with serum-free culture medium. Carboxy- $\mathrm{H}_{2}$ DCFDA $20 \mu \mathrm{M}$ dissolved in serum-free culture medium was then added to each well and the cells were incubated for $1 \mathrm{~h}$ at $37^{\circ} \mathrm{C}$. After this incubation, the medium with the fluorescent dye was replaced with PBS and the plate was incubated for $30 \mathrm{~min}$ at $37^{\circ} \mathrm{C}$. Fluorescence was read at $527 \mathrm{~nm}$, with an excitation wavelength of $495 \mathrm{~nm}$. All experiments were performed four times.
Mitochondrial membrane permeability transition pore measurement. Blockage of the mitochondrial membrane permeability transition pore (mPTP) by compounds was determined with the MitoProbe Transition Assay Kit following the manufacturer's instructions. Briefly, SH-SY5Y human neuroblastoma cells were resuspended in pre-warmed $\mathrm{PBS} / \mathrm{Ca}^{2+}$ buffer at a final concentration of $1 \times 10^{6}$ cells $\mathrm{ml}^{-1}$. Cells were loaded with $0.01 \mu \mathrm{M}$ calcein-AM and incubated at $37^{\circ} \mathrm{C}$ for $15 \mathrm{~min}$. Then, $0.4 \mathrm{mM} \mathrm{CoCl}_{2}$ and compounds at selected concentrations were added and incubated for $15 \mathrm{~min}$ at $37^{\circ} \mathrm{C}$. CsA at $0.2 \mu \mathrm{M}$ was used as positive control. After this incubation, cells were centrifuged and resuspended in $100 \mu \mathrm{l}$ of PBS. Just before analysis, $1 \mathrm{mM}$ tert-butyl hydroperoxide was added to the samples to induce pore opening. Fluorescence intensity was measured at $488 \mathrm{~nm}$ excitation and $517 \mathrm{~nm}$ emission wavelengths by flow cytometry using ImageStreamMKII (Amnis Corporation, Merck-Millipore) and INSPIRE software. The fluorescence of 10,000 events was analysed with IDEAS Application version 6.0 (Amnis Corporation, Merck-Millipore). Experiments were carried out four times.

Reporting summary. Further information on research design is available in the Nature Research Reporting Summary linked to this article.

\section{Data availability}

Crystallographic data for the structure reported in this Article have been deposited at the Cambridge Crystallographic Data Centre under deposition number CCDC $1557733((-)-21 b)$. A copy of the data can be obtained free of charge at https:// www.ccdc.cam.ac.uk/structures/. All other data supporting the findings of this study are available within the Article and its Supplementary Information, or from the corresponding author upon reasonable request.

Received: 22 March 2018; Accepted: 7 February 2019; Published online: 22 March 2019

\section{References}

1. Li, J., Kim, S. G. \& Blenis, J. Rapamycin: one drug, many effects. Cell Metab. 19, 373-379 (2014).

2. Trauner, D. Finding function and form. Nat. Prod. Rep. 31, 411-413 (2014)

3. Wilson, R. M. \& Danishefsky, S. J. Small molecule natural products in the discovery of therapeutic agents: the synthesis connection. J. Org. Chem. 71, 8329-8351 (2006).

4. Wender, P. A. Toward the ideal synthesis and molecular function through synthesis-informed design. Nat. Prod. Rep. 31, 433-440 (2014).

5. Schreiber, S. L. Target-oriented and diversity-oriented organic synthesis in drug discovery. Science 287, 1964-1969 (2000).

6. van Hattum, H. \& Waldmann, H. Biology-oriented synthesis: harnessing the power of evolution. J. Am. Chem. Soc. 136, 11853-11859 (2014).

7. Seiple, I. B. et al. A platform for the discovery of new macrolide antibiotics. Nature 533, 338-345 (2016).

8. Könst, Z. A. et al. Synthesis facilitates an understanding of the structural basis for translation inhibition by the lissoclimides. Nat. Chem. $\mathbf{9}$, 1140-1149 (2017)

9. Bathula, S. R., Akondi, S. M., Mainkar, P. S. \& Chandrasekhar, S. 'Pruning of biomolecules and natural products (PBNP)': an innovative paradigm in drug discovery. Org. Biomol. Chem. 13, 6432-6448 (2015).

10. Yu, M. J., Zheng, W. \& Seletsky, B. M. From micrograms to grams: scale-up synthesis of eribulin mesylate. Nat. Prod. Rep. 30, 1158-1164 (2013).

11. Crane, E. A. \& Gademann, K. Capturing biological activity in natural product fragments by chemical synthesis. Angew. Chem. Int. Ed. 55, 3882-3902 (2016).

12. Romo, D. et al. Evidence for separate binding and scaffolding domains in the immunosuppressive and antitumor marine natural product, pateamine A: design, synthesis and activity studies leading to a potent simplified derivative. J. Am. Chem. Soc. 126, 10582-10588 (2004).

13. Trost, B. The atom economy-a search for synthetic efficiency. Science 254, 1471-1477 (1991)

14. Newhouse, T., Baran, P. S. \& Hoffmann, R. W. The economies of synthesis. Chem. Soc. Rev. 38, 3010-3021 (2009).

15. Young, I. S. \& Baran, P. S. Protecting-group-free synthesis as an opportunity for invention. Nat. Chem. 1, 193-205 (2009).

16. Corey, E. J. \& Cheng, X.-M. The Logic of Chemical Synthesis (Wiley Interscience, New York, NY, 1995).

17. Czakó, B., Kürti, L., Mammoto, A., Ingber, D. E. \& Corey, E. J. Discovery of potent and practical antiangiogenic agents inspired by cortistatin A. J. Am. Chem. Soc. 131, 9014-9019 (2009).

18. Mayol, L., Piccialli, V. \& Sica, D. Gracilin A, an unique: nor-diterpene metabolite from the marine sponge Spongionella gracilis. Tetrahedron Lett. $\mathbf{2 6}$ 1357-1360 (1985).

19. Sanchez, J. A. et al. Identification of Spongionella compounds as cyclosporine A mimics. Pharmacol. Res. 107, 407-414 (2016).

20. Leiros, M. et al. Gracilins: Spongionella-derived promising compounds for Alzheimer disease. Neuropharmacology 93, 285-293 (2015). 
21. Corey, E. J. \& Letavic, M. A. Enantioselective total synthesis of gracilins B and C using catalytic asymmetric Diels-Alder methodology. J. Am. Chem. Soc. 117, 9616-9617 (1995).

22. Rateb, M. E. et al. Bioactive diterpene derivatives from the marine sponge Spongionella sp. J. Nat. Prod. 72, 1471-1476 (2009).

23. Rueda, A. L. A. et al. Cytotoxic bisnorditerpenes from Spongionella pulchella, and the anti-adhesive properties of gracilin B. Lett. Drug Des. Discov. 3, 753-760 (2006).

24. Puliti, R., Fontana, A., Cimino, G., Mattia, C. A. \& Mazzarella, L. Structure of a keto derivative of 9,11-dihydrogracilin A. Acta Crystallogr. C 49, 1373-1376 (1993).

25. Potts, B. C., Faulkner, D. J. \& Jacobs, R. S. Phospholipase A2 inhibitors from marine organisms. J. Nat. Prod. 55, 1701-1717 (1992).

26. Leiros, M. et al. Mitigation of ROS insults by Streptomyces secondary metabolites in primary cortical neurons. ACS Chem. Neurosci. 5, 71-80 (2014).

27. Leiros, M. et al. Spongionella secondary metabolites protect mitochondrial function in cortical neurons against oxidative stress. Mar. Drugs 12, 700-718 (2014).

28. Kofron, J. L., Kuzmic, P., Kishore, V., Colon-Bonilla, E. \& Rich, D. H. Determination of kinetic constants for peptidyl prolyl cis-trans isomerases by an improved spectrophotometric assay. Biochem. 30, 6127-6134 (1991).

29. Walsh, C. T., Zydowsky, L. D. \& McKeon, F. D. Cyclosporin A, the cyclophilin class of peptidylprolyl isomerases, and blockade of $\mathrm{T}$ cell signal transduction. J. Biol. Chem. 267, 13115-13118 (1992).

30. Ferreira, P. A. \& Orry, A. From Drosophila to humans: reflections on the roles of the prolyl isomerases and chaperones, cyclophilins, in cell function and disease. J. Neurogenet. 26, 132-143 (2012).

31. Lee, J. \& Kim, S. S. An overview of cyclophilins in human cancers. J. Int. Med. Res. 38, 1561-1574 (2010).

32. Hogan, P. G., Chen, L., Nardone, J. \& Rao, A. Transcriptional regulation by calcium, calcineurin, and NFAT. Genes Dev. 17, 2205-2232 (2003).

33. Nigro, P., Pompilio, G. \& Capogrossi, M. C. Cyclophilin A: a key player for human disease. Cell Death Dis. 4, e888 (2013).

34. Picone, P., Nuzzo, D., Caruana, L., Scafidi, V. \& Di Carlo, M. Mitochondrial dysfunction: different routes to Alzheimer's disease therapy. Oxid. Med. Cell Longev. 2014, 780179 (2014).

35. Schnermann, M. J. et al. Golgi-modifying properties of macfarlandin E and the synthesis and evaluation of its 2,7-dioxabicyclo[3.2.1] octan-3-one core. Proc. Natl Acad. Sci. USA 107, 6158-6163 (2010).

36. Kornienko, A. \& La Clair, J. J. Covalent modification of biological targets with natural products through Paal-Knorr pyrrole formation. Nat. Prod. Rep. 34, 1051-1060 (2017).

37. Nirmal, N., Praba, G. O. \& Velmurugan, D. Modeling studies on phospholipase $\mathrm{A}_{2}$-inhibitor complexes. Indian J. Biochem. Biophys. 45, 256-262 (2008).

38. Baker, B. J., Kopitzke, R. W., Yoshida, W. Y. \& McClintock, J. B. Chemical and ecological studies of the antarctic sponge dendrilla membranosa. J. Nat. Prod. 58, 1459-1462 (1995).

39. Buckleton, J. S. et al. Structure of tetrahydroaplysulphurin-1. Acta Crystallogr. C 43, 2430-2432 (1987).

40. Abbasov, M. E., Hudson, B. M., Tantillo, D. J. \& Romo, D. Stereodivergent, Diels-Alder-initiated organocascades employing $\alpha, \beta$-unsaturated acylammonium salts: scope, mechanism, and application. Chem. Sci. 8, 1511-1524 (2017).

41. Harvey, N. L. et al. Synthesis of ( \pm )-spongiolactone enabling discovery of a more potent derivative. Chem. Eur. J. 21, 1425-1428 (2015).

42. Burgess, E. M., Penton, H. R., Jr., Taylor, E. A. Synthetic applications of N-carboalkoxysulfamate esters. J. Am. Chem. Soc. 92, 5224-5226 (1970).

43. Alfonso, A. et al. Surface plasmon resonance biosensor method for palytoxin detection based on $\mathrm{Na}^{+}, \mathrm{K}^{+}$-ATPase affinity. Toxins $\mathbf{6}$, 96-107 (2014).

44. Sanchez, J. A. et al. Spongionella secondary metabolites regulate store operated calcium entry modulating mitochondrial functioning in SH-SY5Y neuroblastoma cells. Cell Physiol. Biochem. 37, 779-792 (2015).
45. Damsker, J. M., Bukrinsky, M. I. \& Constant, S. L. Preferential chemotaxis of activated human $\mathrm{CD}^{+} \mathrm{T}$ cells by extracellular cyclophilin A. J. Leukoc. Biol. 82, 613-618 (2007).

46. Moreira, P. I. et al. Mitochondria: a therapeutic target in neurodegeneration. Biochim. Biophys. Acta 1802, 212-220 (2010).

47. Azzolin, L. et al. Antamanide, a derivative of Amanita phalloides, is a novel inhibitor of the mitochondrial permeability transition pore. PLoS One 6, e16280 (2011).

48. Guo, H. X. et al. Novel cyclophilin D inhibitors derived from quinoxaline exhibit highly inhibitory activity against rat mitochondrial swelling and $\mathrm{Ca}^{2+}$ uptake/release. Acta Pharmacol. Sin. 26, 1201-1211 (2005).

49. Rao, V. K., Carlson, E. A. \& Yan, S. S. Mitochondrial permeability transition pore is a potential drug target for neurodegeneration. Biochim. Biophys. Acta 1842, 1267-1272 (2014)

50. Dawar, F. U., Tu, J., Khattak, M. N., Mei, J. \& Lin, L. Cyclophilin A: a key factor in virus replication and potential target for anti-viral therapy. Curr. Issues Mol. Biol. 21, 1-20 (2016).

51. Satoh, K. Cyclophilin A in cardiovascular homeostasis and diseases. Tohoku J. Exp. Med. 235, 1-15 (2015).

52. Abbasov, M. E., Hudson, B. M., Tantillo, D. J. \& Romo, D. Acylammonium salts as dienophiles in Diels-Alder/lactonization organocascades. J. Am. Chem. Soc. 136, 4492-4495 (2014).

53. Halliwell, B. \& Whiteman, M. Measuring reactive species and oxidative damage in vivo and in cell culture: how should you do it and what do the results mean? Br. J. Pharmacol. 142, 231-255 (2004).

\section{Acknowledgements}

The authors acknowledge support from the NIH (R37 GM052964 to D.R.), NSF (CHE1800411, to D.R.) the Robert A. Welch Foundation (AA-1280 to D.R.), FEDER co-funded grants from CONSELLERIA DE Cultura, EDUCACION e ordenación Universitaria Xunta de Galicia (2017 GRC GI-1682, ED431C 2017/01), CDTI and Technological Funds, supported by Ministerio de Economía, Industria y Competitividad (AGL201458210-R, AGL2016-78728-R, AEI/FEDER, UE) (to L.M.B.), ISCIII/PI1/01830 (to A.A.) and RTC-2016-5507-2 and ITC-20161072, from EU POCTEP 0161-Nanoeaters-1-E-1, Interreg AlertoxNet EAPA-317-2016 and H2020 778069-EMERTOX (to L.M.B.) and from the European Union's Seventh Framework Programme managed by the Research Executive Agency (FP7/2007-2013 under grant agreement 312184 PHARMASEA to L.M.B. and M.J.). N. Bhuvanesh and J. Reibenspies (Center for X-ray Analysis, TAMU) secured X-ray data and W. Russell (Laboratory for Biological Mass Spectrometry, TAMU) provided mass data. Correspondence and requests for materials should be directed to D. Romo (chemistry) and L. Botana (biology).

\section{Author contributions}

M.E.A., C.M.C. and M.C. synthesized and characterized all gracilin A derivatives described herein. R.A. and J.A.S. performed the neuroprotection and immunosuppression assays and compiled and wrote the assay data, respectively. L.M.B., E.A. and A.A. designed, analysed and wrote the neuroprotection and immunosuppression assay results and data. D.R. and M.E.A. analysed SARs and wrote the manuscript with input from all authors. M.J. provided samples of the initial lead compound, gracilin A.

\section{Competing interests}

The authors declare no competing interests.

\section{Additional information}

Supplementary information is available for this paper at https://doi.org/10.1038/ s41557-019-0230-0.

Reprints and permissions information is available at www.nature.com/reprints. Correspondence and requests for materials should be addressed to L.M.B. or D.R. Publisher's note: Springer Nature remains neutral with regard to jurisdictional claims in published maps and institutional affiliations.

(c) The Author(s), under exclusive licence to Springer Nature Limited 2019 


\section{natureresearch}

Corresponding author(s): D. Romo \& L. Botana

Last updated by author(s): Jan 10, 2019

\section{Reporting Summary}

Nature Research wishes to improve the reproducibility of the work that we publish. This form provides structure for consistency and transparency in reporting. For further information on Nature Research policies, see Authors \& Referees and the Editorial Policy Checklist.

\section{Statistics}

For all statistical analyses, confirm that the following items are present in the figure legend, table legend, main text, or Methods section.

n/a Confirmed

\The exact sample size $(n)$ for each experimental group/condition, given as a discrete number and unit of measurement

$\bigotimes$ A statement on whether measurements were taken from distinct samples or whether the same sample was measured repeatedly

$\varnothing$ The statistical test(s) used AND whether they are one- or two-sided

Only common tests should be described solely by name; describe more complex techniques in the Methods section.

$\triangle$ A description of all covariates tested

$\square$ A description of any assumptions or corrections, such as tests of normality and adjustment for multiple comparisons

A full description of the statistical parameters including central tendency (e.g. means) or other basic estimates (e.g. regression coefficient)

$\triangle$ AND variation (e.g. standard deviation) or associated estimates of uncertainty (e.g. confidence intervals)

$\$ For null hypothesis testing, the test statistic (e.g. $F, t, r$ ) with confidence intervals, effect sizes, degrees of freedom and $P$ value noted

$\triangle$ Give $P$ values as exact values whenever suitable.

Х $\square$ For Bayesian analysis, information on the choice of priors and Markov chain Monte Carlo settings

Х $\square$ For hierarchical and complex designs, identification of the appropriate level for tests and full reporting of outcomes

Х $\square$ Estimates of effect sizes (e.g. Cohen's $d$, Pearson's $r$ ), indicating how they were calculated

Our web collection on statistics for biologists contains articles on many of the points above.

\section{Software and code}

\section{Policy information about availability of computer code}

Data collection Binding experiments data were collected with Biacore X Control Software from Biacore (GE Healthcare), fluorescence and absorbance data were obtained with Gen5 version 1.07 from Biotek and flow cytometry data with INSPIRE Software from Amnis Corporation (Merck Millipore).

Data analysis

Binding experiments were analyzed with BIAevaluation software version 3.0 from Biacore, flow cytometry data were analyzed with IDEAS Application version 6.0 from Amnis Corporation (Merck Millipore). Fluorescence and absorbance data (MTT, ROS, TMRM, GSH, IL-2 release and CypD and A inhibition assays) were analyzed with GraphPad Prism software version 6.0.

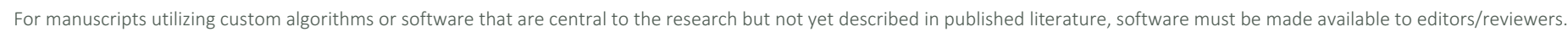
We strongly encourage code deposition in a community repository (e.g. GitHub). See the Nature Research guidelines for submitting code \& software for further information.

\section{Data}

Policy information about availability of data

All manuscripts must include a data availability statement. This statement should provide the following information, where applicable:

- Accession codes, unique identifiers, or web links for publicly available datasets

- A list of figures that have associated raw data

- A description of any restrictions on data availability

The datasets generated during and/or analysed during the current study are available from the corresponding author on reasonable request 
Please select the one below that is the best fit for your research. If you are not sure, read the appropriate sections before making your selection. $\bigotimes$ Life sciences Behavioural \& social sciences Ecological, evolutionary \& environmental sciences

For a reference copy of the document with all sections, see nature.com/documents/nr-reporting-summary-flat.pdf

\section{Life sciences study design}

All studies must disclose on these points even when the disclosure is negative.

Sample size All experiments were repeated a minimum of three times independently (with each condition performed by duplicate). Sample size was chosen based on prior experience of the investigators with similar experiments previously published. The authors have published numerous peer reviewed papers demonstrating clear positive findings with similar sample sizes for the types of experiments included.

Data exclusions Positive controls were added in each experiment, if the positive control did not work, the assay was eliminated. No data points were excluded from analysis in any experiment depicted in this manuscript.

Replication All attempts and replications were successful.

Randomization Randomization is not relevant for cell culture experiments. In the case of human T lymphocytes results, randomization was performed by randomly choosing healthy donors and blinding subject identities to investigators

Blinding Healthy blood donors (human T lymphocytes) were chosen by the medical service of the Universidad de Santiago de Compostela blinding to investigators any information about them. All the data are quantitative, measurements were made using different laboratory instruments such as imagine flow cytometer, biosensor, spectrophotometers, which are not subjected to operator bias.

\section{Reporting for specific materials, systems and methods}

We require information from authors about some types of materials, experimental systems and methods used in many studies. Here, indicate whether each material, system or method listed is relevant to your study. If you are not sure if a list item applies to your research, read the appropriate section before selecting a response.

Materials \& experimental systems

\begin{tabular}{l|l}
\hline $\mathrm{n} / \mathrm{a}$ & Involved in the study \\
$\square$ & $\bigotimes$ Antibodies \\
$\square$ & $\bigotimes$ Eukaryotic cell lines \\
$\square$ & $\square$ Animals and other organisms \\
$\square$ & $\bigotimes$ Human research participants \\
$\bigotimes$ & $\square$ Clinical data
\end{tabular}

\section{Antibodies}

Antibodies used

Validation

\begin{tabular}{l|l}
\multicolumn{2}{l}{ Methods } \\
\hline n/a & Involved in the study \\
$\square$ & $\square$ ChIP-seq \\
$\square$ & $\bigotimes$ Flow cytometry \\
$\square$ & $\square$ MRI-based neuroimaging
\end{tabular}

Х $\square$ MRI-based neuroimaging

Pan T Cell Isolation KIT (Miltenyi Biotec, Catalog number 130-096-535) includes: Pan T Cell Biotin-Antibody Cocktail, human (Cocktail of biotin-conjugated monoclonal antibodies against CD14, CD15, CD16, CD19, CD34, CD36, CD56, CD123, and CD235a (GlycophorinA)) and Pan T Cell MicroBead Cocktail, human (MicroBeads conjugated to monoclonal anti- biotin antibody (isotype: mouse lgG1) and monoclonal anti-CD61 antibody (isotype: mouse lgG1)). Lots: 5150327501 and 5180308452.

Non-target cells, i.e., monocytes, neutrophils, eosinophils, B cells, stem cells, dendritic cells, NK cells, granulocytes, or erythroid cells are labeled by using a cocktail of biotin-conjugated antibodies. The cocktail contains antibodies against CD14, CD15, CD16, CD19, CD34, CD36, CD56, CD123, and CD235a (Glycophorin A). Subsequently, non-target cells are magnetically labelled with the Pan T Cell MicroBead Cocktail. Isolation of highly pure T cells is achieved by depletion of magnetically labelled cells.

\section{Eukaryotic cell lines}

Policy information about cell lines

Cell line source(s)

Human neuroblastoma SH-SY5Y cell line was obtained from American Type Culture Collection, number CRL 2266

Authentication

Cell line was not authenticated aside from authentication provided by ATCC 
Commonly misidentified lines

(See ICLAC register)

\section{Human research participants}

Policy information about studies involving human research participants

Population characteristics

Fresh blood for T lymphocyte isolation was obtained from healthy volunteers. Gender: man and woman. Age: 20 to 40 years old.

Recruitment

Participants were recruited in the medical service of the Universidad de Santiago de Compostela after written consent.

Ethics oversight

The institutional and regional ethical board (Comité Autonómico de Ética da Investigación de Galicia, Comité Territorial de Ética da Investigación de Santiago-Lugo, Secretaria Xeral, Consellería de Sanidade, Xunta de Galicia) approved the study (Reference: 2016/508, Approved date: December 19, 2016, according to the principles outlined in the Declaration of Helsinki).

Note that full information on the approval of the study protocol must also be provided in the manuscript.

\section{Flow Cytometry}

Plots

Confirm that:

$\square$ The axis labels state the marker and fluorochrome used (e.g. CD4-FITC).

$\square$ The axis scales are clearly visible. Include numbers along axes only for bottom left plot of group (a 'group' is an analysis of identical markers).

All plots are contour plots with outliers or pseudocolor plots.

\A numerical value for number of cells or percentage (with statistics) is provided.

\section{Methodology}

Sample preparation

Samples were obtained from human neuroblastoma SH-SY5Y cell line. Cells were resuspended in PBS with calcium and loaded with $0.01 \mu \mathrm{M}$ Calcein-AM and incubated at $37{ }^{\circ} \mathrm{C}$ for $15 \mathrm{~min}$. Then, $0.4 \mathrm{mM} \mathrm{CoCl} 2$ and compounds at selected concentrations were added and incubated for $15 \mathrm{~min}$ at $37 \circ \mathrm{C}$.

Instrument

Image Stream MKII (Amnis Corporation, Merck-Millipore)

Software

Data were collected with INSPIRE Software and analyzed with IDEAS Application version6.0 (Amnis Corporation, Merck-Millipore)

Cell population abundance

One million SH-SY5Y cells were used for each condition. Cell populations were not sorted by primary antibodies, so purity assessment is not relevant

Gating strategy

At first, a scatter plot of the brightfield Area versus Aspect Ratio of the population was performed in order to select single cells and eliminate debris and doublets. Then, a histogram of the brightfield channel Gradient RMS for the population chosen was made to determine focused cells. Cells with a Gradient RMS lower than 40 were excluded from the analysis. Finally, a histogram with the fluorescence intensity of Calcein-AM was performed, all positive cells were selected and the mean was calculated.

Tick this box to confirm that a figure exemplifying the gating strategy is provided in the Supplementary Information. 